\title{
A Novel Method for Seeking Optimal Placement of TCSC to Damp Oscillations in Power Systems
}

\author{
Vandai $\mathrm{Le}^{1,2, *}$, Xinran $\mathrm{Li}^{1}$ and Caoquyen $\mathrm{Le}^{3}$ \\ ${ }^{1}$ College of Electrical and Information Engineering, \\ Hunan University, Hunan, China \\ ${ }^{2}$ Industrial University of Ho Chi Minh City, Viet Nam \\ ${ }^{3}$ Power Engineering Consulting Company 4 (PECC4), \\ Electricity of Vietnam (EVN) \\ ${ }^{1}$ levandai@hui.edu.vn, ${ }^{2}$ lixinran1013@qq.com, ${ }^{3}$ lecaoquyen@gmail.com
}

\begin{abstract}
Thyristor controlled series capacitors (TCSC) damping controller is considered as a useful device to improve the stability of transmission power systems. Seeking the optimal placement of TCSC controller has become a critical issue for the power system stability. From the working point of view, the TCSC controller is used to improve the voltage magnitude, reactive power and oscillation damping of power systems. The effectiveness of the location of TCSC controller, particularly in the damping of power oscillations depends on its location and input signals in the power system during time of grid fault. In this paper, an energetic method based on controllability and observability Gramians in continuous-time is proposed to determine the optimal location of TCSC. The effectiveness of the proposed method has been verified on the 39-bus New England power system.
\end{abstract}

Keywords: controllability and observability Gramians, power system oscillations, TCSC, 39-bus New England power system.

\section{Introduction}

Flexible AC transmission systems (FACTs) are one of the important devices in electrical power systems. Its main function is to optimize power flow along the transmission lines and to improve the damping power oscillations [1]. TCSC is an important FACTs device that is increasingly applied to the modern power systems with long transmission lines. It is used to change the effective reactance of the transmission line, thereby controlling the real power flow in the transmission lines [2]. The existing research has shown that, the effectiveness of TCSC controller depends on its location and input signal.

Most power systems are operating near stability limit, which may result in instability problems. The applications of TCSC have been identified the damping power oscillations and stability enhancement [3-5]. A problem of interest for large power systems is the mitigation of low frequency fluctuations, which often occurs between areas of the interconnected power systems. Frequency response of power system oscillations is usually in the range between 0.1 to $2.0 \mathrm{~Hz}$ depending on the number of generators and can be classified as local and inter-area modes of oscillation. In which the frequency range from 0.7 to $2.0 \mathrm{~Hz}$ is the oscillation frequency of local mode that

* Corresponding Author 
consist of a single generator or a group of generators against the rest of the system. In contrast, oscillations associated with groups of generators, or groups of plants are called inter-area mode having frequency range from 0.1 to $0.8 \mathrm{~Hz}$ of the above frequency range developed in [6]. Power system stabilizers (PSSs) are effective for controlling system oscillations, but they are usually designed for damping local electromechanical oscillations, while the behavior of low frequency inter-area oscillations is generally determined by local parameters of large parts of the power systems [7-8] The FACTs controllers are generally used not only to improve the power system performance but also to damp the power system oscillations [3-4], [9-10]. The problem of designing TCSC controller or choosing the optimal placement of TCSC to improve power system stability improvement has been studied for a long time. However, some of these methods have several drawbacks [5], [7], [10-12].

In the last few years, many researchers have proposed some methods to find the optimal location of TCSC to damp the power system oscillations with only inter-area modes [2], [7], [13]. However, the optimal placement of TCSC controller proposed by these methods does not take effect for transient stability in several operation states. In this study, a novel method based on a maximum energetic approach through controllability and observability Gramians has been proposed for seeking optimal placement of TCSC. The proposed method has been tested in the 39-bus New England power system. The effectiveness of proposed method has been demonstrated through the time domain simulations of several test cases. The first, it is considered the steady-state stability, and then the transient stability is tested to seek optimal placement of the TCSC controller.

The rest of this paper is organized as follows: In section 2, we explain the details for controllability and observability Gramians. In section 3, criteria for seeking optimal placement of TCSC controller is given. In section 4, we discuss the simulation results. Conclusions are drawn in section 5. Finally, in section 6, parameters of the generator, exciters and TCSC are given.

\section{Observability and Controllability Gramians}

\subsection{Gramians Definitions}

In general, power systems are modeled by a set of differential- algebraic equations and can be expressed as:

$$
\begin{aligned}
& \dot{x}(t)=A x(t)+B u(t) \\
& y(t)=C x(t)
\end{aligned}
$$

where $x(t) \in \square^{n}$ is the vector of state variables, $u(t) \in \square^{m}$ and $y(t) \in \square^{r}$ are the vectors of control and measurement variables, respectively, and $A \in \square^{n \times n}, B \in \square^{n \times m}$ and $C \in \square^{r \times n}$ are the state matrix, control matrix and output matrix, respectively. The elements of $A$ and $B$ correspond to the partial derivative of dynamic equations for generators and exciters, and the elements of $C$ correspond to the partial derivative of the algebraic equations with respect to $x(t)$ and $u(t)$, respectively.

Assume that in Equation (1), the vector of state variables of $x(t)$ is described as:

$$
x(t)=\left[\Delta \delta_{1}, \Delta \delta_{2}, \ldots, \Delta \delta_{n}, \Delta \omega_{g 1}, \Delta \omega_{g 2}, \ldots, \Delta \omega_{g n}, z\right]^{T}
$$

where $\Delta \delta_{n}$ and $\Delta \omega_{g n}$ represent the rotor angle and speed of the generator $n$, respectively, and $z$ is the vector of all the other state variables. And the transient controllability and observability expressions of a continuous-time linear system are defined as follows, 
respectively [14]:

$$
\begin{aligned}
& L_{c}(X, T)=\min _{u, x(0)=X} \frac{1}{2} \int_{-T}^{0}\|u(\tau)\|^{2} d \tau, \quad x(-T)=0 \\
& L_{o}(X, T)=\frac{1}{2} \int_{0}^{T}\|y(\tau)\|^{2} d \tau, \quad x(0)=X, u \equiv 0
\end{aligned}
$$

It can be proven that Equation (3) such the transient controllability and observability expressions are:

$$
\begin{aligned}
& L_{c}(X, T)=\frac{1}{2} X^{T} W_{c}-1(T) X \\
& L_{o}(X, T)=\frac{1}{2} X^{T} W_{o}(T) X
\end{aligned}
$$

where $W_{c}(T)=\int_{-T}^{0} e^{A t} B B^{T} e^{A^{T} t} d t, W_{o}(T)=\int_{0}^{T} e^{A^{T} t} C^{T} C e^{A t} d t$ are the transient controllability and observability Gramians on horizon $T$, respectively, $W_{c}(T)$ and $W_{o}(T)$ are the positive definite solutions at time $t=T$, respectively. For the following differential Lyapunov's equations:

$$
\begin{aligned}
& -\dot{W}_{c}(t)+A W_{c}(t)+W_{c}(t) A^{T}=-B B^{T}, W_{c}(0)=0 \\
& -\dot{W}_{o}(t)+A^{T} W_{o}(t)+W_{o}(t) A=-C^{T} C, W_{o}(0)=0
\end{aligned}
$$

If the system in Equation (1) is asymptotically stable around the origin, the controllability expression $\bar{L}_{c}$ and observability expression $\bar{L}_{o}$ are given by:

$$
\begin{aligned}
& \bar{L}_{c}=\min _{u, x(0)=X} \frac{1}{2} \int_{-\infty}^{0}\|u(\tau)\|^{2} d \tau, x(-\infty)=0 \\
& \bar{L}_{o}=\frac{1}{2} \int_{0}^{\infty}\|y(\tau)\|^{2} d \tau, x(0)=X, u \equiv 0
\end{aligned}
$$

When $T \rightarrow \infty: \quad \lim W_{c}(T)=\bar{W}_{c}$ and $\lim W_{o}(T)=\bar{W}_{o}$, then $\bar{W}_{c}$ and $\bar{W}_{o}$ are obtained as the unique positive definite solution for the following Lyapunov's equations:

$$
\begin{aligned}
& A W_{c}+W_{c} A^{T}+B B^{T}=0 \\
& A^{T} W_{o}+W_{o} A+C^{T} C=0
\end{aligned}
$$

Since the controllability matrix $W_{c}$ depends on the control input matrix $B$, the control energy can be affected by choosing this control input matrix, while the observability matrix $W_{o}$ depends on the output signal matrix $C$, the output energy can be affected by choosing this output signal matrix. Furthermore, when the system is only stable and detectable (even some non-controllable and non-observable, are stable state variables), then Equation (5) shows that, the Gramians contain only some non-negative matrices, since the singular values corresponding to the non-controllable or non-observable states are equal to zero [15].

\subsection{Gramians Properties}

The different energetic approaches for positioning actuators and sensors location from the linear dynamic model addressed by literature [16]. First, in the case of transient disturbance, it must be imposed by a final state variable at final time while minimizing the input energy. This is equivalent to maximizing the norm of the controllability matrix $W_{c}$. The second point of view corresponds to maximize the total energy transmitted from the actuators to the structure for a given input; this is equivalent to maximizing the trace of $W_{c}$. The third 
approach is a geometrical interpretation of Gramian matrix. If the system is controllable, $W_{c}$ is a symmetric positive matrix associated with quadratic form, i.e., to ellipsoids whose axis directions are given by eigenvector of $W_{c}$ and lengths proportional to eigenvalues. The bigger the ellipsoid is, the more controllable the system is, and this is equivalent to maximizing the Gramian eigenvalues.

The notion of Gramian is related to the energy $E_{c}$ needed to move the system at any state $x$ from the origin [15]:

$$
E_{c}=x^{T} W_{c} x=\sum_{i=1}^{n} \sigma_{i} x^{T} v_{i} v_{i}^{T} x
$$

Equation (8) is easily tractable with classical algebra and can be describes as:

$$
\operatorname{trace}\left(W_{c}\right)=\sum_{i=1}^{n} \sigma_{i}
$$

where $\sigma_{i}$ and $v_{i}$ are the $i^{\text {th }}$ singular value and the normalized $(\|v\|=1) i^{\text {th }}$ singular vector, respectively.

The singular value decomposition represents a spatial decomposition of the energy contained in the impulse response. Each singular value represents the contribution in term of energy, at a particular direction. The sum of the singular values represents the contribution to the total energy [17-19].

The TCSC controller has been placed on the basis of the maximization of the total energy and so that Equation (9) can be used as an optimization criterion for optimal control input selection.

\section{Criteria for Optimal Placement of TCSC Controller}

The main idea of this method is to select the TCSC controller corresponding to the minimum energy to control the most significant modes and to select the placement of TCSC controller, in order to improve the small signal stability as well as the transient stability of the power system.

The problem is to find out the control input signal which drives the state variables to make the system behaves in the desired way. The power system is investigated with perturbation of the active power of transmission lines. Several critical perturbations of active power lines were considered and the maximum value of energy was calculated. In Figure 1, the input signals are local active power where TCSC is connected.

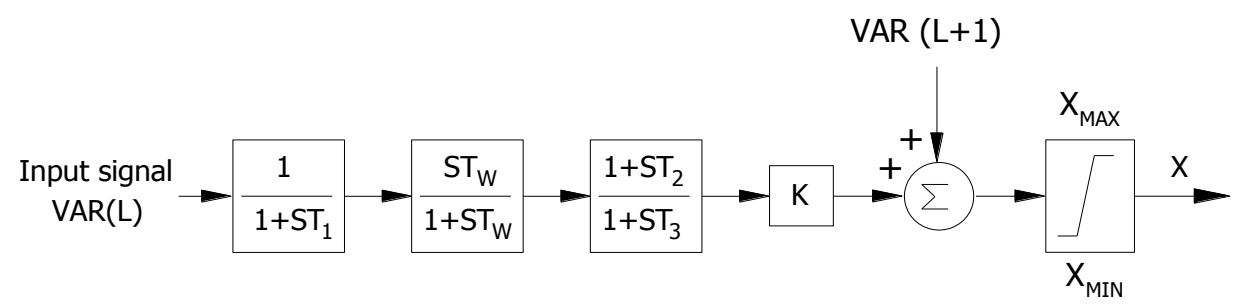

\section{Figure 1. Block Diagram of "CRANI" TCSC Model in PSS/E Dynamic Library}

In this paper, an algorithm for seeking an optimal placement of TCSC to prevent power system oscillation is proposed and described as below:

1. With a placement of TCSC $((i=1,2, \ldots, n)$, generating a set of $m$ independent scenario, $(j=1,2, \ldots, m)$ (with $m$ : large enough). In scenarios $j$, the control input $\alpha_{j}$ (perturbation of 
active power of transmission line) is analyzed. Calculate matrices $A_{i j}, B_{i j}, C_{i j}$. Unstable equilibrium cases are eliminated by checking stable conditions of characteristic matrix $A_{i j}$.

2. For each scenarios $j$, calculate the controllability Gramian: $W_{c}^{i j}$, which satisfies one of the Lyapunov's equations

$$
A_{i j} W_{c}^{i j}+W_{c}^{i j} A_{i j}^{T}+B_{i j} B_{i j}^{T}=0
$$

3. Calculate the individual energy by a norm corresponding to the control inputs as following

$$
E_{i j}=\operatorname{trace}\left(W_{c}^{i j}\right)
$$

4. The optimal selection of FACTs is chosen by basing on the total maximum energy over all scenarios that is calculated by

$$
\underset{i=1, \ldots, n}{\operatorname{Max}}\left[E_{j}=\sum_{j=i}^{m} E_{i j}\right]
$$

\section{Simulation Results}

In order to illustrate of the proposed method, the well-known New England power system benchmark was considered. This system consists of 10 generators and 39 buses, with generator 2 taken as reference generator. The single-line diagram and the result of load flow analysis are given in Figure 2.

All of the scenarios for the perturbation of active power are considered in Table 1 and controllability indices for disturbance of active power are given in Table 2

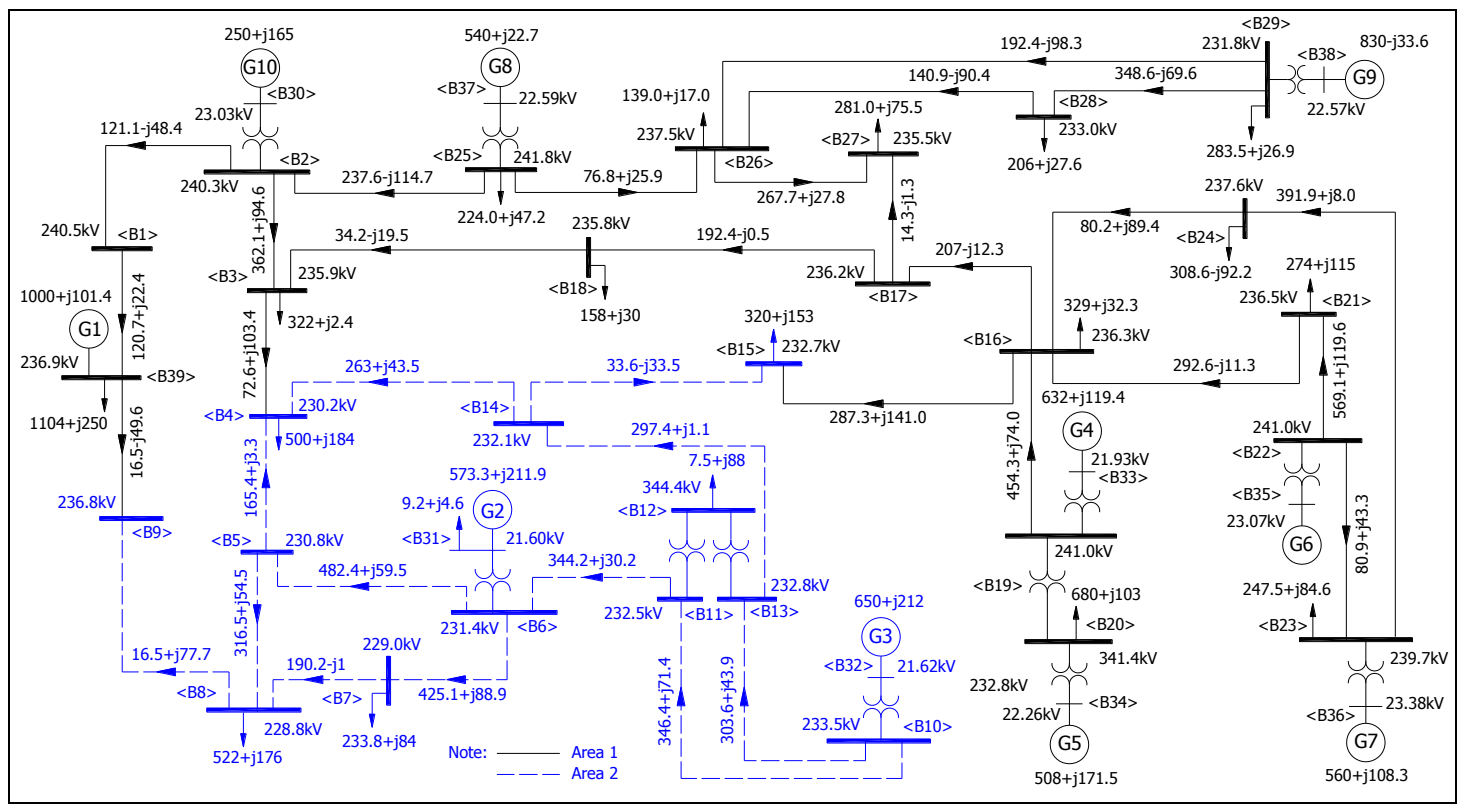

Figure 2. The Result of Load Flow Analysis on the 39-bus New England Systems 
Table 1. Scenarios for Studying

\begin{tabular}{cc}
\hline Ranking & The disturbance of lines \\
\hline 1 & Line $4-14$ \\
2 & Line $17-18$ \\
3 & Line $25-26$ \\
4 & Line $27-26$ \\
5 & Line $14-15$ \\
\hline
\end{tabular}

Program Power System Simulation Engineering (PSS/E) [8] is employed to analyze small signal and transient stability. In this paper, We assume that all generators are modeled by Round Rotor Generator (quadratic saturation) - GENROU, all excitation systems are identical, and modeled by IEEE Static Excitation System - EXST1, which are modeled by standard models in the PSS/E library.

Table 2 shows that the TCSC controller placed in the line 16-17 has the highest total energy and almost the highest energy for different input signals of disturbance of active power on the other lines, which are listed in Table 1. Therefore the line 16-17 is an optimal location to place the TCSC controller.

In order to evaluate the effectiveness of the TCSC controller for damping oscillations, several dynamic simulation cases on the 39-bus New England power system along with the TCSC controller are carried out to analyze.

In this test, we considered the three-phase fault on lines that occurs the nearest bus and this line is outage at $0.35 \mathrm{sec}$, to confirm the effect of the location of TCSC controller on power system through the damping oscillations of rotor angle of generator and active power on transmission line in the shortest time possible. Some of contingency cases in the order of tests are listed in Table 3.

Table 2. Energy Value According to TCSC Locations

\begin{tabular}{|c|c|c|c|c|c|c|c|}
\hline \multirow{2}{*}{ No } & \multirow{2}{*}{$\begin{array}{l}\text { TCSC on } \\
\text { line }\end{array}$} & \multicolumn{5}{|c|}{ The disturbance of lines $\left(\times 10^{3}\right)$} & \multirow{2}{*}{$\begin{array}{c}\text { Energy SUM } \\
W_{c} * 10^{3}\end{array}$} \\
\hline & & $4-14$ & $17-18$ & $25-26$ & $27-26$ & 14-15 & \\
\hline 1 & $16-17$ & 8.460 & 17.23 & 5.920 & 12.43 & 11.160 & 55.200 \\
\hline 2 & $28-29$ & 0.330 & 1.470 & 6.690 & 6.940 & 0.890 & 16.320 \\
\hline 4 & $16-19$ & 5.530 & 13.42 & 3.950 & 9.730 & 10.810 & 43.440 \\
\hline 5 & $16-21$ & 1.007 & 2.487 & 0.811 & 1.952 & 2.826 & 9.0830 \\
\hline 6 & $21-22$ & 6.520 & 1.328 & 3.770 & 9.350 & 10.470 & 31.438 \\
\hline 7 & $28-26$ & 0.101 & 0.265 & 1.029 & 0.931 & 0.209 & 2.5350 \\
\hline 8 & $17-27$ & 0.569 & 0.657 & 1.782 & 3.142 & 0.823 & 6.9730 \\
\hline 9 & $26-29$ & 1.450 & 3.920 & 15.24 & 11.42 & 2.790 & 34.820 \\
\hline 10 & $23-24$ & 1.199 & 2.941 & 0.938 & 2.240 & 3.306 & 10.624 \\
\hline 11 & $1-39$ & 0.287 & 0.862 & 1.312 & 1.103 & 1.082 & 4.6460 \\
\hline
\end{tabular}


Figure 3 shows that the response of active power oscillations in the line 17-18 when a three phase fault is simulated in the line 15-16 (nearby bus 16) at $1 \mathrm{sec}$ and this line is outage after $0.35 \mathrm{sec}$, with and without TCSC controller placed in the line 16-17. It can be observed that the active power oscillations are damped out in about $8 \mathrm{sec}$ when TCSC controller is used in the power system, which is more effective than when the TCSC controller is not used in the power system.

Figure 4 shows that the response of output equivalent reactance oscillations on the line 16-17 where TCSC controller is located to correspond with active power input signals in the one, when a three-phase fault is simulated in the line 15-16 (nearby bus 16) at $1 \mathrm{sec}$ and this line is outage after $0.35 \mathrm{sec}$. The variation of the equivalent reactance is limited to $0.002225 \mathrm{pu}$ to $0.01068 \mathrm{pu}$ corresponding to the maximum and minimum amplitudes of active power oscillations of the line 16-17, respectively. In other words, when the amplitude of active power reaches the maximum value, the TCSC controller supplies the minimum equivalent reactance of $0.75 X_{16-17}$, whereas when the amplitude of active power sinks to the minimum value, the TCSC controller supplies the maximum equivalent reactance of 1.2 $X_{16-17}$, where $X_{16-17}$ is the reactance of transmission line 16-17.

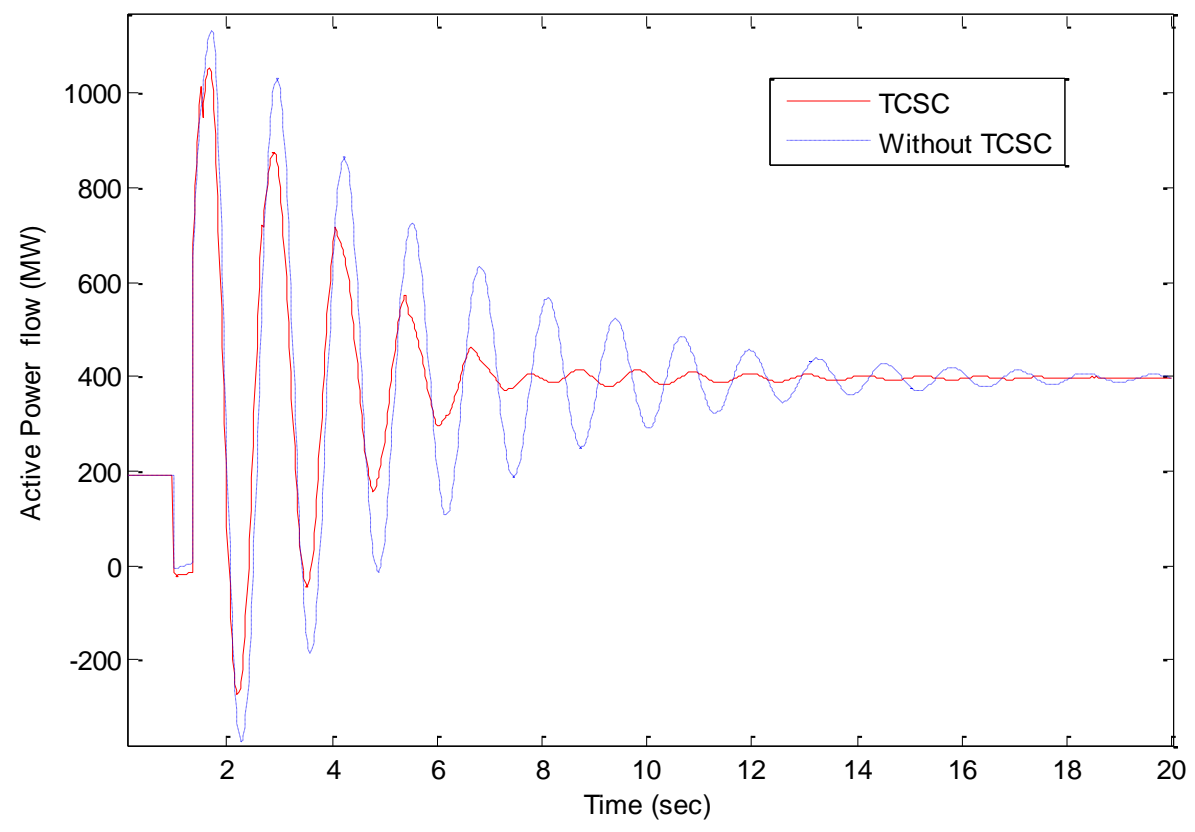

Figure 3. Active Power Variation of the Line 17-18 for a Three-phase Fault to Ranking 1, with or without the TCSC Controller in the Line 16-17 


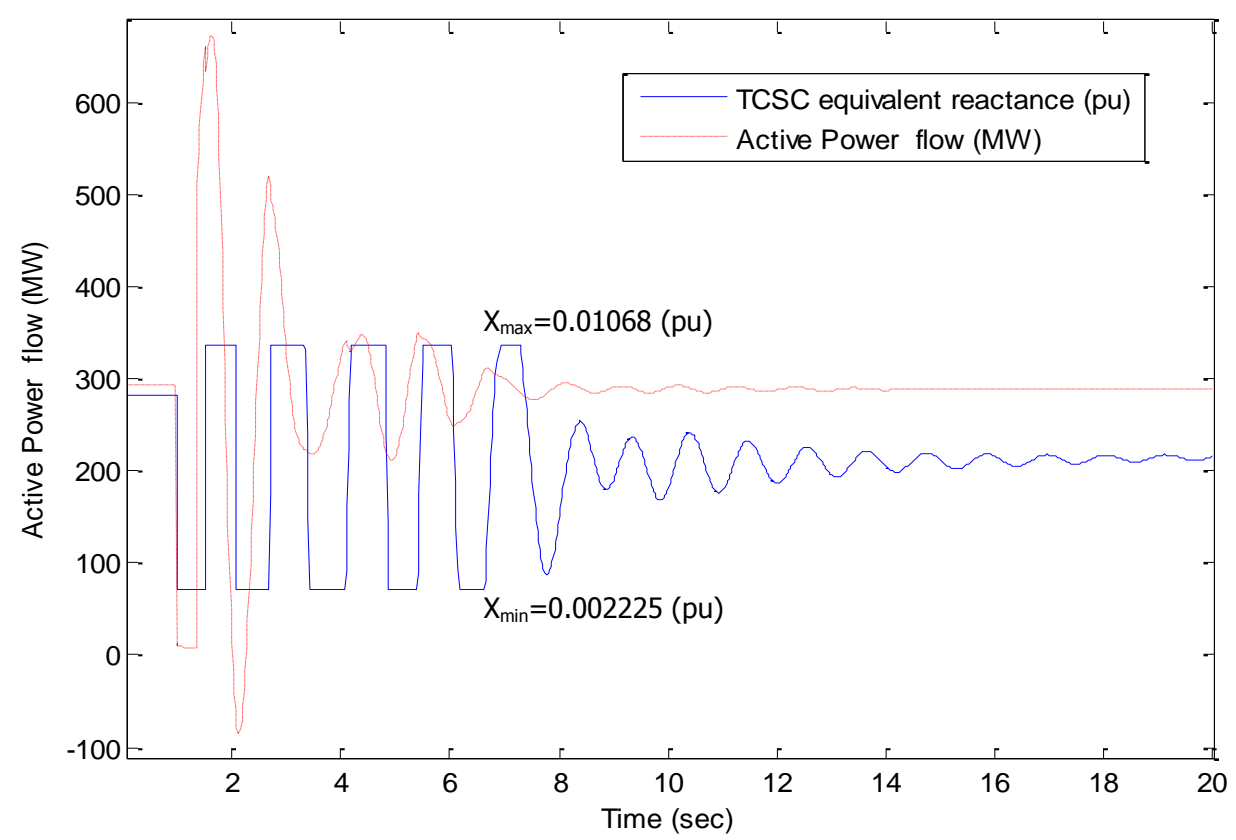

Figure 4. The Equivalent Reactance of TCSC Controller in the Line 16-17 to Correspond with Active Power Flow Variation, when a Three-phase Fault Simulated in the Line 15-16

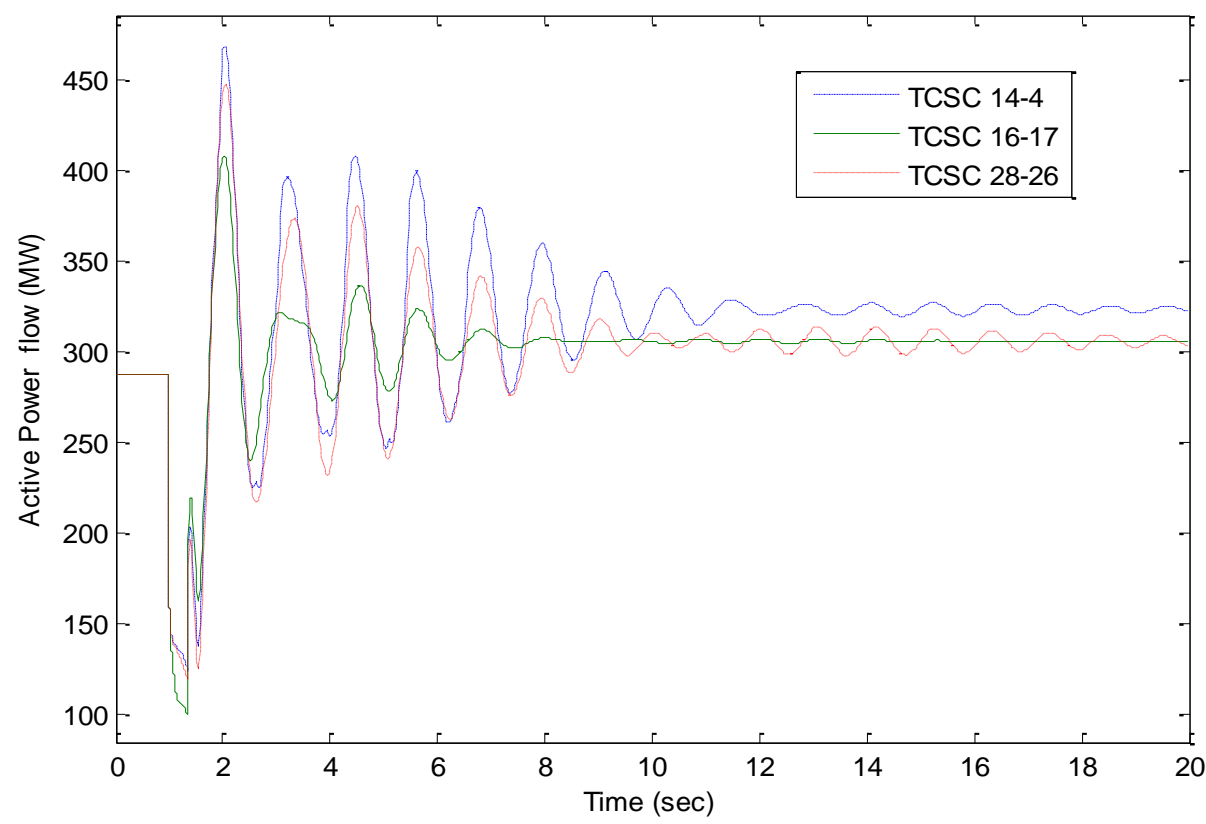

Figure 5. Active Power Variation of Line 15-16 for a Three-phase Fault to Ranking 2, the TCSC Controller on Line 16-17, 26-28 and 14-4 


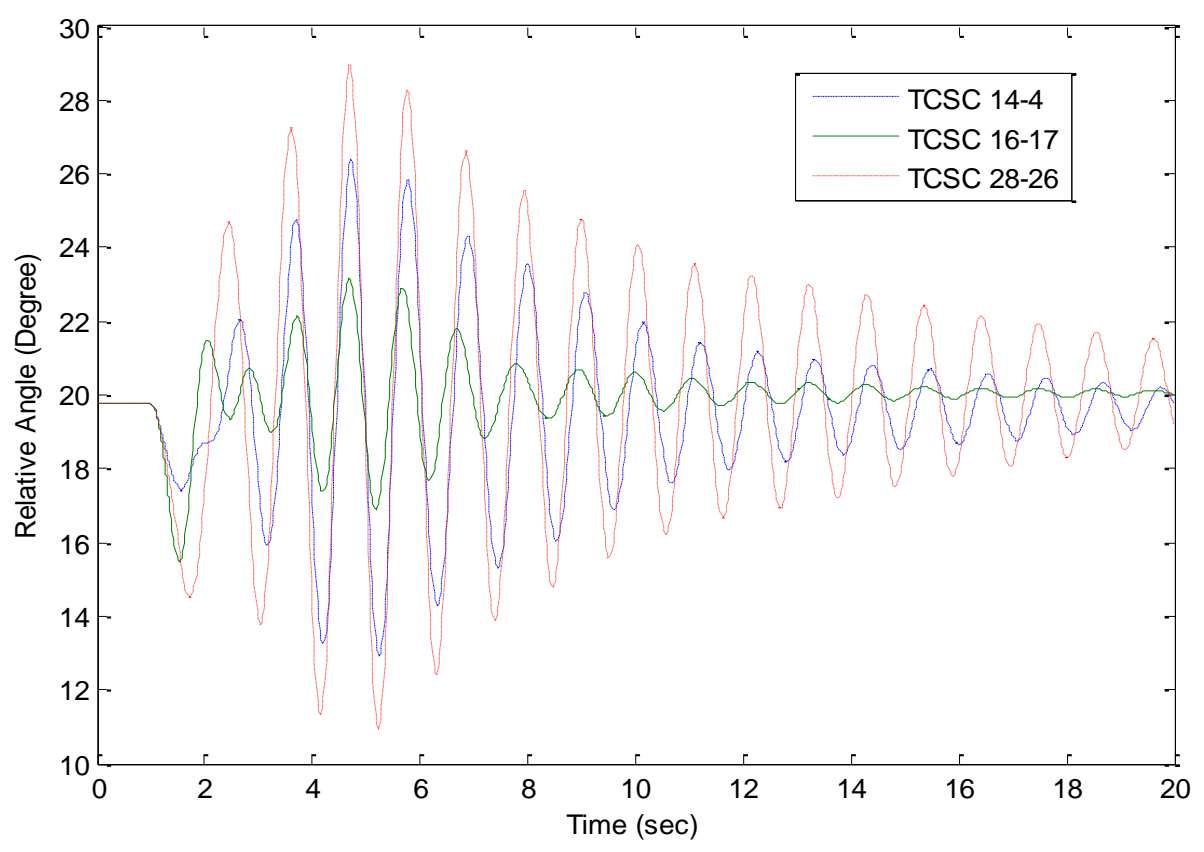

Figure 6. Rotor Angle Variation of Generator G1 for a Three-phase Fault to Ranking 2, the TCSC Controller Placed in Line 16-17, 26-28 and 14-4

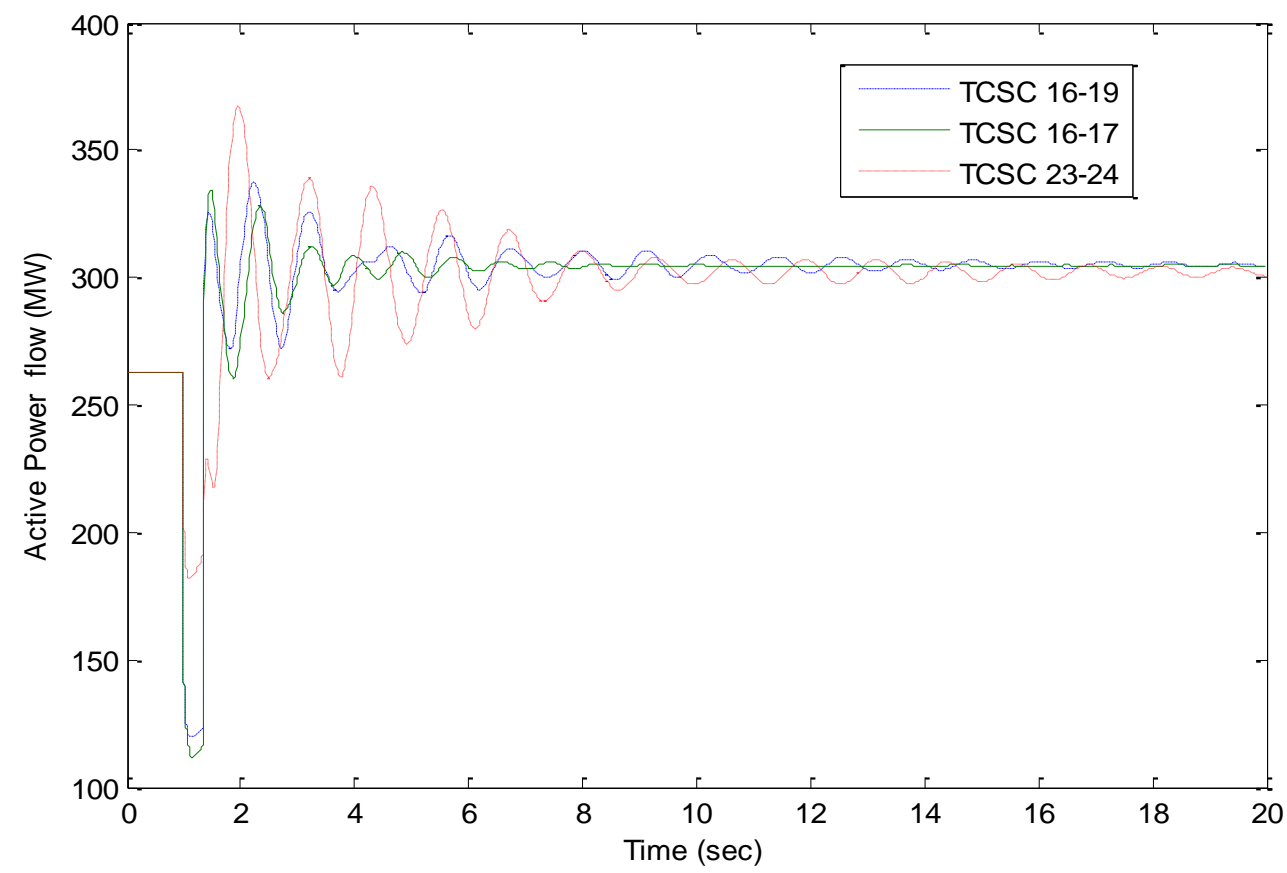

Figure 7. Active Power Variation of Line 4-14 for a Three-phase Fault to Ranking 3, the TCSC Placed in Lines 16-17, 16-19 and 23-24 
International Journal of Control and Automation

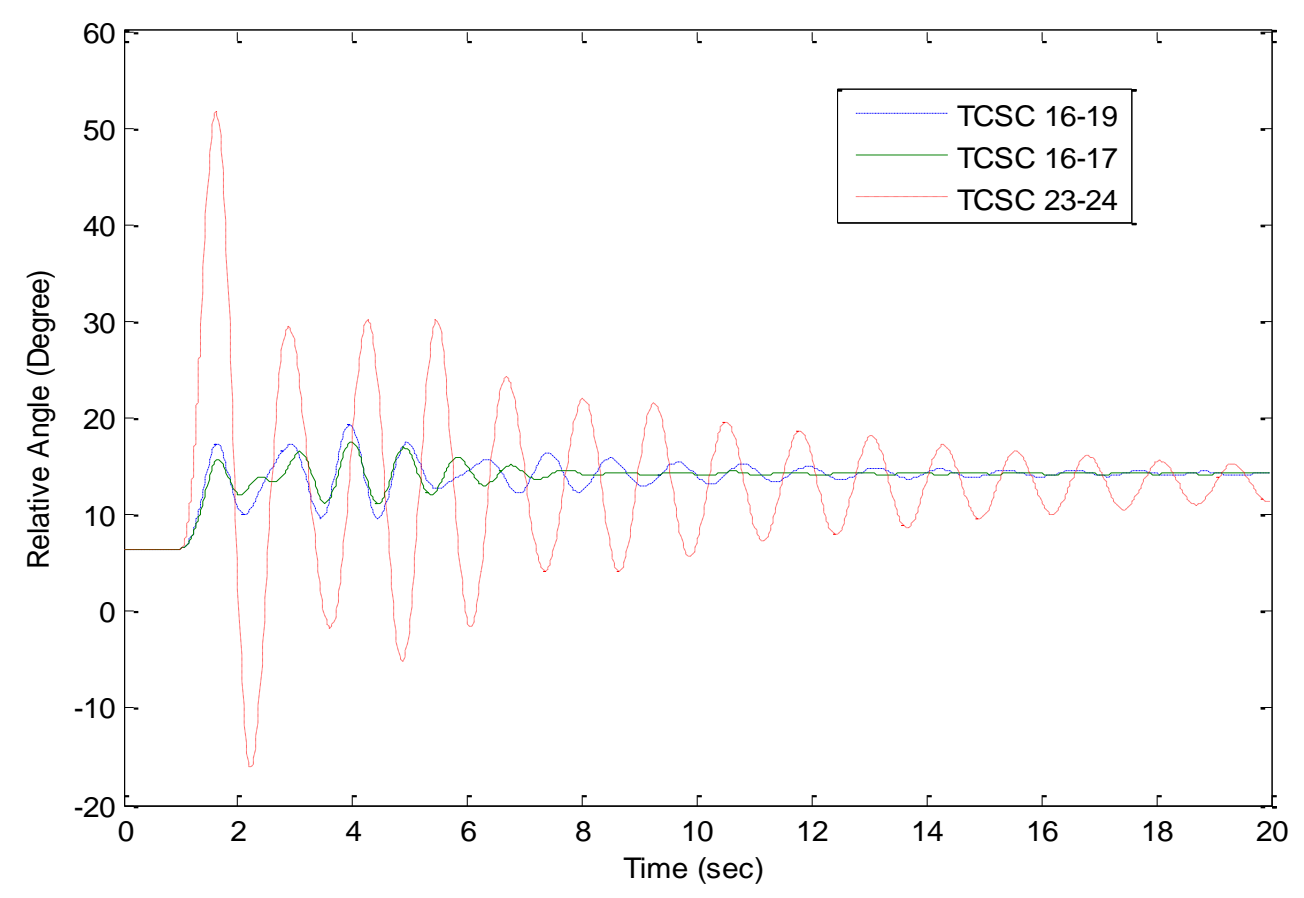

Figure 8. Rotor angle Variation of Generator G8 for a Three-phase Fault to Ranking 2, the TCSC Controller Placed in Line 16-17, 16-19 and 23-24

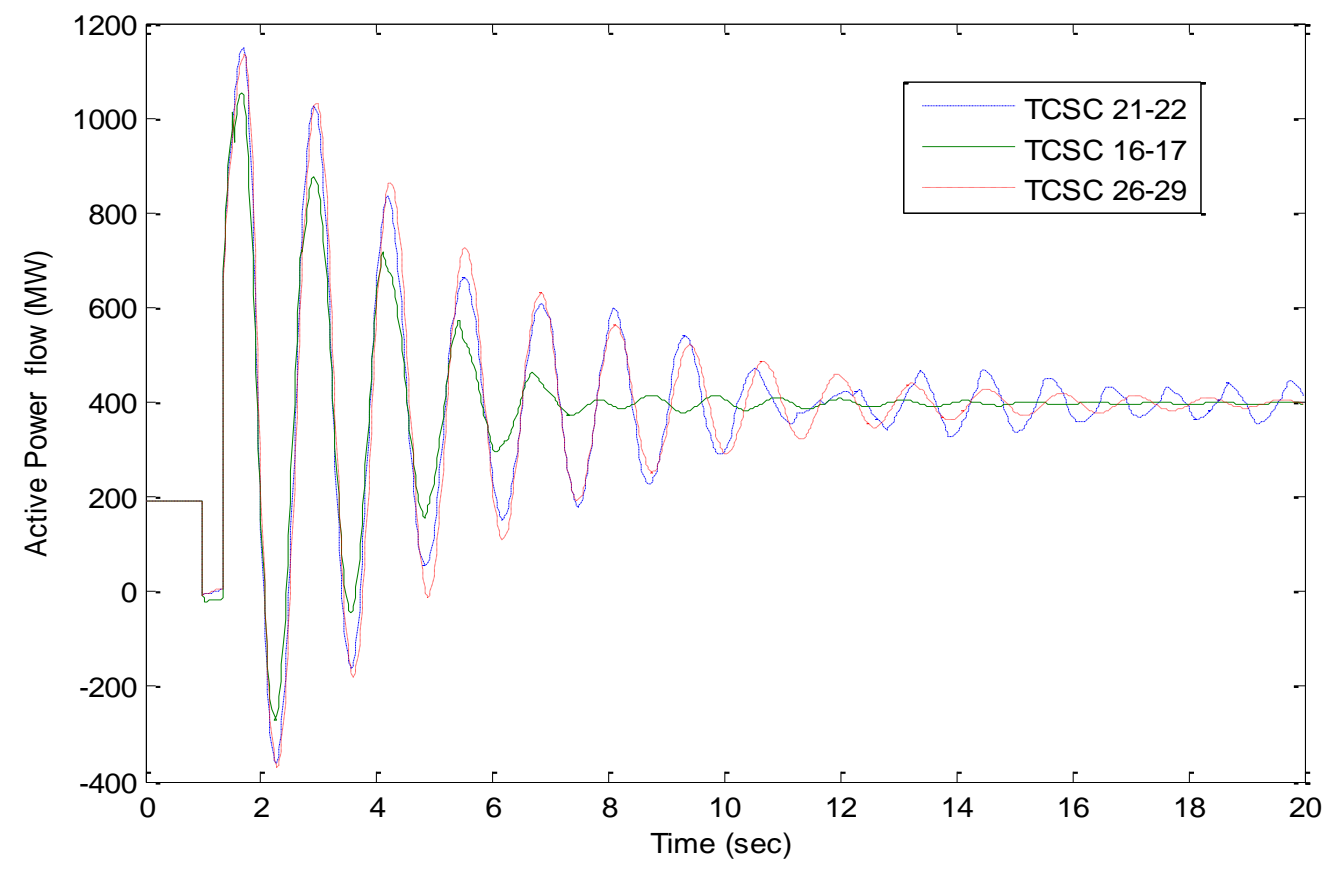

Figure 9. Active Power Variation of 17-18 Line for a Three-phase Fault to Ranking 4, the TCSC Controller Placed in Line 16-17, 21-22 and 26-29 


\section{Table 3. Contingency Ranking with Transient Stability Test}

\begin{tabular}{ccc}
\hline Ranking & $\begin{array}{c}\text { Fault is } \\
\text { nearby bus }\end{array}$ & $\begin{array}{c}\text { Line outage after } \\
0.35 \mathrm{sec}\end{array}$ \\
\hline 1 & Bus 16 & Line 15-16 \\
2 & Bus 3 & Line 3-18 \\
3 & Bus 3 & Line 2-3 \\
4 & Bus 17 & Line 17-27 \\
\hline
\end{tabular}

Figures 5, 7 and 9 give the active power flows of lines 15-16, 4-14 and 17-18, the TCSC controller located in line (14-4, 16-17 and 28-26), line (16-19, 16-17 and 23-24), and line (21-22, 16-17 and 26-29), after a three-phase-fault in lines 3-18, 2-3 and 17-27 occurs at $1 \mathrm{sec}$, respectively. It is clearly observed that the active power oscillations of transmission line with the TCSC controller located in line 16-17 damped out in $7 \mathrm{sec}$, while other cases continue to be kept oscillating.

Figures 6 and 8 give the rotor angle variation of generators G1 and G8, the TCSC controller located in line (16-17, 26-28 and 14-4), and line (16-17, 16-19, and 23-24), after the three phase fault happened on line 3-18 at $1 \mathrm{sec}$, respectively. It is clearly observed that the oscillations of rotor angle with the TCSC controller located in line 16-17 damped out in 8 sec, while other cases continue to be kept oscillating.

It can be seen that with the placement of TCSC controller respectively located in line 16-17, 16-19 and 26-29, the damping oscillation improves significantly. However, the TCSC controller located the line16-17 for the system damping is the best location since its energy is the highest compared with other locations. It also can be seen that oscillations are damped out when the TCSC controller is placed in line 16-17, which is more effective compared with other locations.

\section{Conclusions}

The controllability and observability Gramians method for seeking optimal placement of TCSC is proposed in this paper. This method is carried out in the 39-bus New England power systems, and the simulation results confirm the effective damping performance. These index values of energy have been computed for different input signal of TCSC controller and the best location of the TCSC controller is in the line with maximum energy.

The simulation results show that the active power variation of lines and the rotor angle variation of generators are significantly improved in the damping of oscillations, when TCSC controller placed in the line 16-17 where is the best location found using the proposed method. This method can be used in large-scale power systems.

\section{Appendix}

Dynamic parameters in per unit on its rating are given below:

Generators:

$X_{d}=1.8, X_{q}=1.7, X_{l}=1.2, X_{d}^{\prime}=0.3, X_{q}^{\prime}=0.55, X_{d}^{\prime \prime}=0.25, X_{q}^{\prime \prime}=0.25 R_{A}=0.0025$, $T_{d o}^{\prime}=8.0, T_{q o}^{\prime}=0.4, T_{d o}^{\prime \prime}=0.03, T_{q o}^{\prime \prime}=0.05, H=6.5, S(1.0)=0.0377, S(1,2)=0.1821$.

Exciters: 
$T_{A}=0.01, T_{B}=1, T_{C}=1, T_{R}=0.01 \quad K_{A}=200, V_{R M A X}=6.4, V_{R M I N}=-6, K_{C}=0, K_{F}=0$,
$T_{F}=1$.

TCSC:

\begin{tabular}{c|c|c|c|c|c|c|c}
\hline Model & $\mathrm{T}_{1}$ & $\mathrm{~T}_{2}$ & $\mathrm{~T}_{3}$ & $\mathrm{~T}_{\mathrm{W}}$ & $\mathrm{K}$ & $\mathrm{X}_{\max }$ & $\mathrm{X}_{\min }$ \\
CRANI & 0.1 & 0.1 & 0.4 & 100 & 0.75 & $1.2 * \mathrm{X}$ & $0.25 * \mathrm{X}$ \\
\hline
\end{tabular}

Note: $\mathrm{X}$ is the reactance of transmission line

\section{Acknowledgment}

The authors sincerely acknowledge the financial support provided by the National Natural Science Foundation of China (NSFC) Changsha, China under project no. 751202093 for carrying out the present research work.

\section{References}

[1] P. K. Dash, S. Morris and S. Mishra, "Design of a nonlinear variable gain fuzzy controller for Facts devices", Control Systems Technology, IEEE Transmissions, vol. 12, no. 3, (2004), pp. 428-438.

[2] B. Kalyan Kumar, S. N. Singh and S. C. Srivastava, "Placement of FACTS controllers using modal controllability indices to damp out power system oscillations", Generation, Transmission \& Distribution, IET, vol. 1, no. 2, (2007), pp. 209-217.

[3] E. S. Ali and S. M. A. Elazim, "TCSC damping controller design based on bacteria foraging optimization algorithm for a multimachine power system", Electrical Power and Energy Systems, vol. 37, no. 1, (2012), pp. 20-30.

[4] H. Shayeghi, A. Safari and H. A. Shayanfar, "PSS and TCSC damping controller coordinated design using PSO in multi-machine power system", Energy Conversion and Management, vol. 51, no. 12, (2010), pp. 2930-2937.

[5] P. S. Dolan, J. R. Smith and W. A. Mittelstadt, "A study of TCSC optimal damping control parameters for pifferent operating conditions", Power Systems, IEEE Transmissions, vol. 10, no. 4, (1995), pp. 1972-1978.

[6] M. Klein, G. J. Rogers and P. Kundur, "A fundamental study of inter-area oscillations in power systems", Power Systems, IEEE Transactions on, vol. 6, no. 3, (1991), pp. 914-921.

[7] M. W. Mustafa and N. Magaji, "Optimal location of static var compensator device for damping oscillations", American Journal of Engineering and Applied Sciences, vol. 2, no. 2, (2009), pp. 353-359.

[8] Siemens PTI, PSS/E 30.2 Progrm Operational Manual, vol. 2, (2005).

[9] W. Du, X. Wu, H. F. Wang and R. Dunn, "Feasibility study to damp power system multi-mode oscillations by using a single FACTS device", Journal of Electrical Power \& Energy Systems, vol. 32, no. 6, (2010), pp. 645-655.

[10] J. Machowski, S. Robak and J. Bialek, "Damping of power swings by optimal control of series compensators", Power System Automation and Control, Proceedings of the $10^{\text {th }}$ International Conference, (1997).

[11] A. D. Del Rosso, C. A. Canizres and V. M. Dona, "A study of TCSC controller design for power system stability improvement", Power Systems, IEEE Transactions, vol. 18, no. 4, (2003), pp. 1487-1496.

[12] N. Yang, Q. Liu and J. D. McCalley, "TCSC controller design for damping interarea oscillations", Power Systems, IEEE Transactions, vol. 13, no. 4, (1998), pp. 1304-1310.

[13] N. Magaji and M. W. Musatafa, "Optimal location of TCSC device for damping oscillations", Journal of Engineering and Applied Sciences, ARPN, vol. 4, no. 3, (2009), pp. 28-34.

[14] E. Kreindler and P. E. Sarachik, "On the concept of controllability and observability of linear systems", Automatic and Control, IEEE Transactions, vol. 9, no. 2, (2003), pp. 129-136.

[15] N. D. Toan, D. Georges and T. Q. Tuan, "An energy approach to optimal selection of controllers/sensors in power system", International Journal of Emerging Electric Power Systems, vol. 9, no. 6, (2008), pp. 1-19.

[16] S. Leleu, H. A. Kandil and Y. Bonnassieux, "Piezoelectric actuators and sensors location for active control of flexible structures", Instrumentation and Measurement, IEEE Transactions, vol. 50, no. 6, (2001), pp. $1577-1588$.

[17] M. A. Wicks and R. A. DeCarlo, "An energy approach to controllability", Decision and Control, Proceeding of the 27th IEEE Conference, (1988). 
[18] H. Juergen and F. Edgar Thomsas, "An improved method for nonlinear model reduction using balancing of empirical gramians", Computer \& Chemical Engineering, vol. 26, (2002), pp. 1379-1397.

[19] B. C. Moore, "Principal component analysis in linear systems: Controllability, observability, and model reduction", Automatic and Control, IEEE Transactions, vol. 26, no. 1, (1981), pp. 17-32
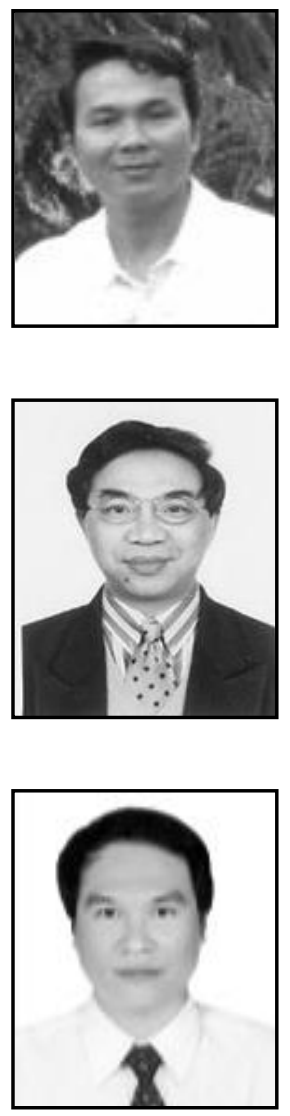

\section{Authors}

Vandai Le, he received MSc degree in Electrical Engineering from the Ho Chi Minh City University of Technology, Vietnam in 2008. He is currently a Ph.D. student at Hunan University, Changsha, China. He is a lecturer with the Industry University of Ho Chi Minh City, Viet Nam. His research interests are in stability and control of FACTs, wind power generation, HVDC and grid integration.

Xinran Li, he received the B.Sc. degree from the College of Electrical and Information engineering, Hunan University, Changsha, China, in 1981 and the M.Sc. and Ph.D. degrees from North China Electric Power University, Baoding, China, in 1988 and 1999, respectively. Currently, he is a Full Professor with Hunan University, Hunan, China. His research interests are in power system load modeling, voltage stability and voltage control.

Caoquyen Le, he received the B.Sc. and M.Sc. degrees from the Ho Chi Minh City University of Technology, Vietnam in 1999 and 2005, respectively. He received his Ph.D. degree in Electrical Engineering from the Danang University in 2012. Since 2011 he has been working as Director of Substation engineering Centre in the Power Engineering Consulting Company - PECC4. His research interests are in power stability and voltage control. 
International Journal of Control and Automation Vol. 8, No.4 (2015) 